\title{
ELECTRONIC COLLOQUIA
}

Besides the scientific discussion on crucial topics and notes with demonstrations of ongoing projects, two topics suggesting perspectives on our future and our past will be addressed by panels during COLING-90.

Invitations to participate by supplying brief comments as e-mail messages, at any time up to and including the conference session period were distributed. We here summarize what has been said in the electronic colloquia so far. A fuller publication will be prepared after the conference.

The topics selected for COLING-90 were

* The Unfinished Language

* Homunculus Loquens 